\title{
Measurement of urinary hippuric and m-methylhippuric acids by gas chromatography
}

\author{
J.P. BUCHET and R. R. LAUWERYS \\ Industrial and Medical Toxicology Unit, Louvain University, avenue Chapelle-aux-Champs 4, \\ 1200 Brussels, Belgium
}

\begin{abstract}
Buchet, J. P., and Lauwerys, R. R. (1973). British Journal of Industrial Medicine, 30, 125-128. Measurement of urinary hippuric and $m$-methylhippuric acids by gas chromatography. A gas chromatographic method for the determination of hippuric and m-methylhippuric acids in urine is described. A known amount of heptadecanoic acid (internal standard) is added to the urine before its extraction with ethyl acetate. After evaporation of the solvent, the acids are methylated with diazomethane. The residue is taken up in methanol and injected into the gas chromatograph. The ratio of the height of the hippuric or m-methylhippuric acid peak to the height of the heptadecanoic acid peak is calculated and by reference to a calibration curve prepared in the same conditions the urine concentration of the two acids is determined.
\end{abstract}

Several investigators (Pagnotto and Lieberman, 1967; Ikeda and Ohtsuji, 1969; Ogata, Tomokuni, and Takatsuka, 1970) have demonstrated that exposure to toluene and $\mathrm{m}$ - or $\mathrm{p}$-xylene can be estimated by measuring the amount of hippuric and methyhippuric acids excreted in urine.

Spectrophotometric procedures have been developed for the determination of these metabolites (Gaffney, Schreier, DiFerrante, and Altman, 1954; Ogata, Sugiyama, and Moriyasu, 1962; Pagnotto and Lieberman, 1967; Ogata, Tomokuni, and Takatsuka, 1969). The ultraviolet absorption method described by Pagnotto and Lieberman (1967) for hippuric acid measurement offers the advantage of simplicity but lacks specificity.

Ogata et al. (1969) have described a specific method for the quantitative determination of hippuric and $\mathrm{m}$ - or $\mathrm{p}$-methylhippuric acids in urine. However, their procedure, which is a modification of Gaffney's paper chromatographic method (Gaffney et al., 1954), is too elaborate and time-consuming for the routine monitoring of workers exposed to toluene and $\mathrm{m}$ - or p-xylene. It involves quantitative extraction of acidified urine with ethyl acetate or ethyl ether/ethanol $(9: 1)$, thin-layer or paper chromatography of the extract, elution of the hippuric and methylhippuric acid spots with ethanol before or after their conversion to their azlactones, and spectrophotometric measurement of the latter.

The purpose of this paper is to describe a gas chromotographic procedure which is as specific and sensitive as Ogata's method and offers the advantage of being more rapid. Furthermore, the use of an internal standard gives the gas chromatographic technique a high degree of precision.

\section{Materials and methods}

\section{Reagents}

Ethyl acetate, petroleum ether $\left(40-60^{\circ} \mathrm{C}\right)$, diethyl ether, diisopropyl ether, methanol, ethanol, p-dimethylaminobenzaldehyde, acetic anhydride, totylsulphonyl methylnitrosamide, heptadecanoic acid, and hippuric acid were purchased from Merck (Darmstadt, Germany); m-toluoyl chloride was obtained from Aldrich-Europe (Belgium). An ethereal solution of diazomethane was prepared from tolylsulphonyl methylnitrosamide as described by Vogel (1956).

m-Methylhippuric acid was synthesized by following 
Vogel's procedure for hippuric acid synthesis except that benzoyl chloride was replaced by $\mathrm{m}$-toluoyl chloride.

Urine treatment

Transfer $0.2 \mathrm{ml}$ of urine, $0.2 \mathrm{ml}$ of the internal standard solution (heptadecanoic acid $1 \mathrm{mg} / \mathrm{ml}$ in ethanol), and $0.1 \mathrm{ml}$ of $\mathrm{HCl} 0.5 \mathrm{~N}$ and $3.5 \mathrm{ml}$ of ethyl acetate successively into a glass stoppered tube. Shake the tube for 2 minutes in an Analis (Belgium) mechanical shaker; allow it to stand for $\mathbf{1 0}$ minutes. Remove approximately $3 \mathrm{ml}$ of the upper organic phase containing hippuric and methylhippuric acids and the internal standard and evaporate to dryness under a stream of nitrogen. The methylation of the acids is performed by addition of $0.5 \mathrm{ml}$ of a methanol-diethyl ether mixture $(10 / 90 \mathrm{v} / \mathrm{v})$ and a sufficient volume of the ethereal solution of diazomethane until the yellow colour persists.

Allow to stand for 10 minutes, evaporate the solvent, and dissolve the residue in 0.1 to $0.2 \mathrm{ml}$ of methanol before injection into the gas chromatograph.

Fifty to one hundred urine samples are usually treated at the same time.

\section{Apparatus}

The determination was performed with a Varian (type 1800) gas chromatograph equipped with two columns. The analysis column consisted of $100 \mathrm{~cm}$ long stainless steel tubing (internal diameter $1 / 8$ inch) filled with Chromosorb W(AW-DMCS) (80/100 mesh) coated with $3 \%$ SE30. The oven was maintained at a temperature of $160^{\circ} \mathrm{C}$ and the injectors and detectors at $250^{\circ} \mathrm{C}$.

Nitrogen used as the carrier gas $(40 \mathrm{ml} / \mathrm{min})$ was dried by passing it through a Packard gas dryer. The flame of each of the ionization detectors was supplied with air $(300 \mathrm{ml} / \mathrm{min})$ and hydrogen $(60 \mathrm{ml} / \mathrm{min})$. The electrometer range was set to $10^{-10} \mathrm{amp} / \mathrm{mV}$ position and the attenuator switch between 4 and 16 according to the amount of hippuric and methylhippuric acids present in the sample injected (1-2 $\mu \mathrm{l})$.

Each of the detectors was connected to a pen recorder Philips model P.M. 8000 (chart speed : $10 \mathrm{~mm} / \mathrm{min}$ ).

Calculation of hippuric and m-methylhippuric acid concentration

The ratio of the height of the hippuric or m-methylhippuric acid peak to the height of the heptadecanoic acid peak was calculated and, by reference to a standard curve (see results), the urine concentration of the two metabolites was determined and expressed in milligrammes/ litre of urine or milligrammes/gramme creatinine. The creatinine content of urine was measured by Jaffe's technique (Henry, 1965) using a Carlo Erba Colorimeter Laboratory Analyzer, model CLA 1510.

\section{Results}

Elution pattern of the methyl esters of hippuric, m-methylhippuric, and heptadecanoic acids

An example of a chromatogram is shown in Figure 1. The three methylesters are well separated and are eluted within 8 minutes after the injection. We found that to get accurate results with urine, the injection solvent selected is an important factor. Petroleum ether and diethyl ether, for example, are



FIG. 1. Gas chromatographic elution pattern of the three acids extracted from water and urine.

not suitable. Although they dissolve the purified esters they are unable to solubilize completely the residue obtained from urine and therefore give erratic results. Reproducible results are obtained with methanol.

Calibration curve of hippuric and m-methylhippuric acids in water and in urine

Standard aqueous solutions of hippuric and m-methylhippuric acids were prepared, ranging from 1 to $5 \mathrm{mg} / \mathrm{ml} ; 0.2 \mathrm{ml}$ of each solution was added to an equal volume of water or control urine and the analysis was performed as described above. Figure 2 shows that a linear relationship was obtained between the peak height ratios and the hippuric or $\mathrm{m}$ methylhippuric acid concentrations. The calibration curve of hippuric acid in urine parallels that in water; the displacement of the line for urine from that for water is due to the hippuric acid normally present in urine of a person non-occupationally exposed to toluene.

Since control urine does not contain m-methylhippuric acid, the calibration curves for this compound in water and in urine are identical (Fig. 2B). 

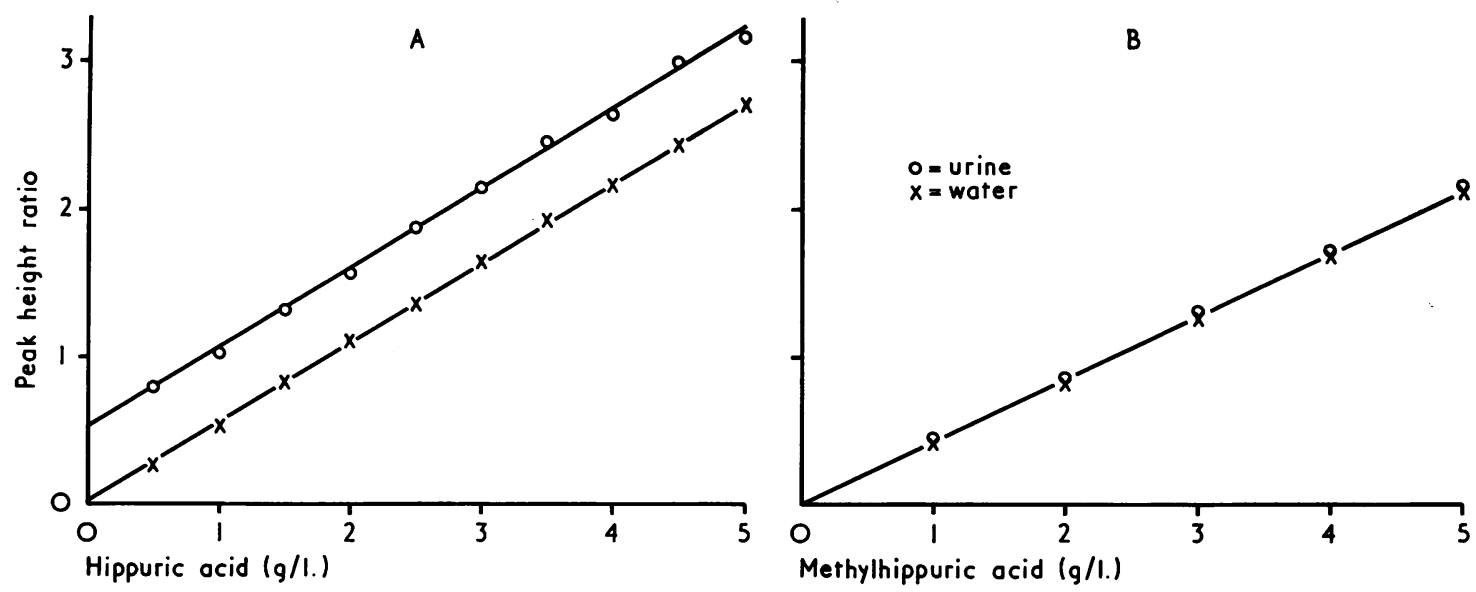

FIG. 2. Calibration curves of hippuric acid (A) and m-methylhippuric acid (B) in water and in urine.

The results demonstrate that the extraction efficiency of the two metabolites is the same from urine and water. Their determination in urine can therefore be made by reference to a standard curve based on the peak height ratios in water.

The day to day variability of the standard curve obtained on the same column is small; the coefficient of variation of the slope (6 determinations) is less than $3 \%$. However, as a routine control of the experimental conditions at least three points of the standard curve were run at the same time during each series of urine analyses. Several extraction solvents (ethyl acetate, heptane, hexane, petroleum ether, diisopropyl ether) have been tested. The least variability was obtained with ethyl acetate, probably due to its constant extraction efficiency for the internal standard and the hippuric and methylhippuric acids over a large range of concentration of these two metabolites. However, because an internal standard is used it is not necessary to get a quantitative extraction of the three substances. A single extraction step is therefore sufficient, which is an important advantage over the spectrophotometric techniques. The effect of urinary $\mathrm{pH}$ on the reproducibility and efficiency of the extraction was also investigated. We found that the degree of extraction is constant over $\mathrm{HCl}$ concentrations ranging from 0.05 to $0.5 \mathrm{~N}$.

\section{Comparison of colorimetric and gas chromatographic methods}

The hippuric acid content of five different control urines was determined by gas chromatography and by the colorimetric technique of Ogata et al. (1969) (p-dimethylaminobenzaldehyde solution in acetic anhydride was used to form the azlactone). As shown in the Table, the results obtained by the two techniques are not statistically different. It should, however, be noticed that the colorimetric technique cannot be used for the simultaneous determination of hippuric and methylhippuric acids in urine without a preliminary chromatography on paper or silica gel.

Hippuric and m-methylhippuric acids content of normal urine

The urine of 31 persons with no occupational exposure to solvents was examined. No m-methylhippuric acid was detected. The mean hippuric acid concentration \pm SEM was $1 \cdot 10 \pm 0.15 \mathrm{~g} / 1$ or $0.80 \pm 0.08 \mathrm{~g} / \mathrm{g}$ creatinine. We also found that urine can be stored at $4^{\circ} \mathrm{C}$ under tartaric acid $(0.1 \%)$ without any loss of activity.

\section{TABLE}

Determination of Hippuric Acid in Normal Urine By Colorimetry aNd Gas ChromatoGRAPHY

\begin{tabular}{c|c|c|c}
\hline \multirow{3}{*}{$\begin{array}{c}\text { Subject } \\
\text { No. }\end{array}$} & \multicolumn{2}{|c}{ Hippuric acid concentration $(g / l)$} \\
\cline { 2 - 4 } & $\begin{array}{c}\text { Gas chromatography } \\
(G C)\end{array}$ & $\begin{array}{c}\text { Colorimetry } \\
(C)\end{array}$ & $\begin{array}{c}\text { Difference } \\
\text { GC }-C\end{array}$ \\
\hline 1 & 0.970 & 0.880 & $+0 \cdot 090$ \\
2 & 0.940 & 1.040 & -0.100 \\
3 & 1.250 & 1.250 & 0 \\
4 & 5.800 & 5.620 & $+0 \cdot 180$ \\
5 & 0.950 & 0.840 & $+0 \cdot 110$ \\
\hline
\end{tabular}

$P>0 \cdot 10$ (paired $t$ test) 


\section{Discussion}

Our investigation clearly demonstrates that the gas liquid chromatographic method offers many advantages over the current spectrophotometric techniques for the determination of hippuric and m-methylhippuric acids in urine.

It is as specific and precise as the elaborate colorimetric technique of Ogata et al. (1969) and one extraction of urine is sufficient. The use of an internal standard increases the speed of the analysis and reduces greatly the probability of error, and as a consequence of the use of an internal standard, quantitative recovery of the metabolites is not an absolute condition. Furthermore, except for the first two volume measurements (urine and internal standard), the manipulations require less meticulous attention than the spectrophotometric technique since the parameter sought is a ratio of two signals (metabolite - internal standard) and not their absolute values.

The sensitivity of the gas chromatographic technique is also adequate; it allows exact evaluation of the hippuric acid content of urine of persons not exposed to toluene. This procedure can therefore be used routinely for the control of workers exposed to toluene and xylene.

We would like to thank Mr J. M. Defeld for his valuable technical assistance.

\section{References}

Gaffney, G. W., Schreier, K., DiFerrante, N., and Altman, K. I. (1954). The quantitative determination of hippuric acid. Journal of Biological Chemistry, 206, 695-698.

Henry, R. J. (1965). Clinical Chemistry-Principles and Technics. Harper and Row, London.

Ikeda, M., and Ohtsuji, H. (1969). Significance of urinary hippuric acid determination as an index of toluene exposure. British Journal of Industrial Medicine, 26, 244-246.

Ogata, M., Sugiyama, K., and Moriyasu, H. (1962). Studies on poisoning. IV. Toluene concentration in air and urinary hippuric acid measured by paperchromatography and mass screening examination method. Acta medicinae Okayama, 16, 283-292.

- Tomokuni, K., and Takatsuka, Y. (1969). Quantitative determination in urine of hippuric acid and $m$ - or $p$ methylhippuric acid, metabolites of toluene and $\mathrm{m}$ - or p-xylene. British Journal of Industrial Medicine, 26, 330-334.

,$---(1970)$. Urinary excretion of hippuric acid and $\mathrm{m}$ - or $\mathrm{p}$-methylhippuric acid in the urine of persons exposed to vapours of toluene and $\mathrm{m}$ - or $\mathrm{p}$-xylene as a test of exposure. British Journal of Industrial Medicine, 27, 43-50.

Pagnotto, L. D., and Lieberman, L. M. (1967). Urinary hippuric acid excretion as an index of toluene exposure. American Industrial Hygiene Association Journal, 28, 129-134.

Vogel, A. I. (1956). A Text-book of Practical Organic Chemistry, 3rd ed. Longmans, London.

Received for publication June 5, 1972. 